\title{
Frequent serial fecal corticoid measures from rats reflect circadian and ovarian corticosterone rhythms
}

\author{
S A Cavigelli ${ }^{1}$, S L Monfort ${ }^{2}$, T K Whitney ${ }^{3}$, Y S Mechref ${ }^{4}$, \\ M Novotny ${ }^{4}$ and M K McClintock ${ }^{5}$ \\ ${ }^{1}$ Department of Biobehavioral Health, Pennsylvania State University, State College, PA 16802, USA \\ ${ }^{2}$ National Zoo's Conservation \& Research Center, Smithsonian Institution, Front Royal, VA 22630, USA \\ ${ }^{3}$ Department of Psychology, University of Wisconsin, Madison, WI 53706, USA \\ ${ }^{4}$ Department of Chemistry, Indiana University, Bloomington, IN 47405, USA \\ ${ }^{5}$ Department of Psychology and the Institute for Mind \& Biology, University of Chicago, Chicago, IL 60637, USA \\ (Requests for offprints should be addressed to S A Cavigelli; Email: s-cavigelli@psu.edu)
}

\begin{abstract}
The circadian glucocorticoid rhythm provides important information on the functioning of the hypothalamicpituitary-adrenal axis in individuals. Frequent repeated blood sampling can limit the kinds of studies conducted on this rhythm, particularly in small laboratory rodents that have limited blood volumes and are easily stressed by handling. We developed an extraction and assay protocol to measure fecal corticosterone metabolites in repeated samples collected from undisturbed male and female adult Sprague-Dawley rats. This fecal measure provides a noninvasive method to assess changes in corticosterone within a single animal over time, with sufficient temporal acuity
\end{abstract}

to quantify several characteristics of the circadian rhythm: e.g. the nadir, acrophase, and asymmetry (saw-tooth) of the rhythm. Males excreted more immunoreactive fecal corticoids than did females. Across the estrous cycle, females produced more fecal corticoids on proestrus (the day of the preovulatory luteinizing hormone surge) than during estrus or metestrus. These results establish a baseline from which to study environmental, psychological, and physiological disturbances of the circadian corticosterone rhythm within individual rats.

Journal of Endocrinology (2005) 184, 153-163

\section{Introduction}

The hypothalamic-pituitary-adrenal axis is exquisitely sensitive to environmental perturbations. Given this highly adaptive sensitivity, measuring hypothalamicpituitary-adrenal axis function in undisturbed subjects can be problematic, particularly in small laboratory rodents. The simple act of handling, restraining, or collecting a blood sample from the tail vein in small rodents can significantly elevate glucocorticoid levels within minutes (Smith \& Gala 1977, Tuli et al. 1995, Haemisch et al. 1999). Thus, even mildly invasive repeated sampling limits studies on the temporal secretory dynamics of the hypothalamic-pituitary-adrenal axis as they normally occur in the absence of human contact. The circadian glucocorticoid rhythm is altered in several pathological states; e.g. major depressive disorder (Sachar et al. 1973, Linkowski et al. 1985, Pfohl et al. 1985), Alzheimer's disease, sleep deprivation (Spiegel et al. 1999), and normal aging (van Cauter et al. 1996, Kern et al. 1996). Identifying such disruptions in a rodent model can be difficult given the problems involved in measuring the glucocorticoid rhythm within an individual over time. As a means of assessing daily temporal dynamics of corticosterone production non-invasively, we have applied newly developed techniques for measuring corticosteroid metabolites in feces to document continuous corticosterone production over time in minimally disturbed male and female laboratory rats.

In small rodents with limited blood volumes, fecal pellets are particularly effective for measuring the cirdadian rhythms of hormones. In contrast to larger mammals, which defecate only once or twice a day, small rodents defecate several pellets every 1-2 h (Kishibayashi et al. 1995), affording the higher temporal resolution needed to detect circadian rhythms and perturbations by a stressor. Fecal corticoid measures are also effective for documenting changes in glucocorticoid production over days if not months. Small rodents have small blood volumes, thereby limiting the frequency, volume, and/or duration with which blood samples can be collected for repeated measures within a single individual. Furthermore, jugular cannulation, which is typically used for repeated blood sampling, has been associated with high failure and complication rates after a week (Gans \& McClintock 1993). In addition, the cannulation is a stressor, necessarily limiting 
an animal's mobility and activity levels, and altering normal glucocorticoid levels and circadian rhythms (Royo et al. 2004). Finally, fecal pellets have relatively high concentrations of corticosteroid metabolites (e.g. male SpragueDawley rats excrete $80 \%$ of exogenously administered radiolabeled corticosterone in the feces (Bamberg et al. 2001)), and recent research has shown that a single antibody cross-reacts with a broad spectrum of corticosteroid metabolites, and therefore can be useful in a host of species (Goymann et al. 1999, Wasser et al. 2000), including rodents (Harper \& Austad 2000, Ponzio et al. 2004).

In a first study, we assessed the functionality of fecal corticoid sampling by quantifying the temporal characteristics of its circadian rhythm and comparing them with those established from blood samples. Most mammalian species experience a marked circadian rhythm in circulating glucocorticoid concentrations, with peak circulating concentrations in the order of 5-10 times more concentrated than trough levels, and occurring just prior to the daily active period, i.e. in the early morning for diurnal species and at the end of the light period in nocturnal species. In many mammalian species, including rats, this rhythm is asymmetrical: circulating glucocorticoid concentrations rise rapidly at the end of the inactive sleep period, peak just prior to the active period, and then levels slowly decrease across the day to the nadir at the end of the active period (e.g. Guilleman et al. 1959, Retiene et al. 1968, Krieger 1973, Dahl et al. 1991, Frank et al. 1995, Rohatagi et al. 1996, Atkinson \& Waddell 1997).

In a second study, we determined if the fecal corticoid measure could detect changes in glucocorticoid production across the estrous cycle in female rats. Female corticosterone production is highest on proestrus (prior to ovulation) and drops to its minimum during estrus and metestrus (Atkinson \& Waddell 1997). On proestrus, the circadian peak in circulating glucocorticoid concentrations is twice that of estrus or metestrus.

The third study compared male and female rat fecal corticoid production. Males and females differ in liver function, produce a different array of fecal corticosterone metabolites, maintain different levels of corticosterone and corticosteroid-binding globulin in circulation, have differential plasma corticosterone-binding capacity, and metabolize corticosterone at different rates (Gala \& Westphal 1965, Eriksson \& Gustafsson 1970, Ottenweller et al. 1979, Woodward et al. 1991). Given these large sex differences, we expected sex differences in corticoid metabolite excretion based on HPLC co-chromatography and liquid chromatography-mass spectrometry (LC-MS) analyses.

\section{Methods}

Animals

We collected fecal samples from young adult SpragueDawley rats: 10 adult males at 15 weeks of age and nine adult females at 11 weeks of age. All animals were housed singly in a reversed $14 \mathrm{~h}$ light $/ 10 \mathrm{~h}$ darkness cycle with the dark phase beginning at 10:00 h (central daylight saving time, CDST). Temperature in the animal rooms was maintained at $21 \pm 1{ }^{\circ} \mathrm{C}$ with 15 air changes per hour and food (Harlan Teklad Rodent Diet W, no. 8604) and water provided ad libitum. All animals lived in hanging cages $(20 \times 24 \times 18 \mathrm{~cm})$ with wire bottoms through which their feces dropped into a pan of standard wood-shaving bedding (Sani-Chips, laboratory grade). Animals had lived alone in these cages for more than 1 month at the time of this study.

Estrous-cycle days were monitored in females by measuring estrogen and progesterone metabolites in feces. Proestrus was defined as the day of maximal estrogen and progesterone metabolite excretion and these days were verified with vaginal cytology data (LeFevre \& McClintock 1988).

\section{Sample collection}

Samples were collected for a full day from males to characterize the circadian rhythm, and across 4 days for females to characterize changes across the estrous cycle. Rats' cages did not have to be opened or disturbed during sample collection, thereby minimizing possible disruption of the corticoid rhythm. Samples were collected with forceps directly from the bedding under each hanging cage, and wetness (urine) surrounding any sample was noted. Collections occurred seven times a day at 6:00, 9:00, 12:00, 15:00, 18:00, 21:00, and 24:00 h. This sampling frequency was developed to maximize temporal acuity while maximizing the probability that at least one pellet would be defecated per sampling interval (Kishibayashi et al. 1995). Given that samples collected at 9:00 h could be defecated any time between 6:00 and 9:00 h CDST, we identified each sampling interval according to the midpoint time of the interval (e.g. samples collected at 9:00 h were identified as coming from the 7:30 $\mathrm{h}$ interval). The dark-phase samples (10:30, 13:30, 16:30, and 19:30 h intervals) occurred during the rats' active period, and the light-phase samples (22:30, 3:00, and 7:30 h intervals) occurred during the rats' inactive period. Samples were collected into Whirl-Pak bags (Nasco, Fort Atkinson, WI, USA), labeled, and stored at $-30{ }^{\circ} \mathrm{C}$ until extracted. If a sample was collected from a urine-soaked area of bedding, the sample bag was marked as 'urine-contaminated'.

\section{Fecal corticoid extraction}

Fecal steroids were extracted using previously published methods (Wasser et al. 1994). Briefly, frozen samples were thawed, dried overnight in a centrifugal evaporator, crushed into a dust-like material, and $0 \cdot 2 \mathrm{~g}$ weighed into 
a $15 \mathrm{ml}$ centrifuge tube. Ethanol $10 \mathrm{ml}(100 \%)$ was added to each sample which was then boiled in a water bath for 20 min. Upon removal from the bath, tubes were centrifuged for $15 \mathrm{~min}$ and the supernatant was poured off into a glass tube. Ethanol $5 \mathrm{ml}$ was added to the fecal sample tube, and the sample was then vortexed for $1 \mathrm{~min}$, re-centrifuged for $15 \mathrm{~min}$, and the supernatant added to the previous $10 \mathrm{ml}$ of extract. Supernatants were evaporated under air then re-constituted with $1 \mathrm{ml}$ methanol and stored at $-80{ }^{\circ} \mathrm{C}$ until assay. To monitor procedural losses, radiolabeled corticosterone (1200 c.p.m.) was added to a subset of samples before extraction and post-extraction recovery was quantified by counting a portion of the extractant. Hormone mass was corrected by dividing by the percentage of isotope recovery.

To control for daily fluctuations in fecal mass, we present total fecal corticoids produced during each collection interval as opposed to the more traditional method of presenting concentration of corticoids in fecal samples. Thus, hormone concentrations were expressed as the total mass (ng) excreted per 3-h sampling interval. If a rat defecated $0.5 \mathrm{~g}$ fecal matter (dried) in a given 3-h interval, and the concentration of corticoids in that sample was $100 \mathrm{ng} / \mathrm{g}$ of dry fecal matter, then the total corticoid mass for that sampling interval would be $50 \mathrm{ng}$. Likewise, if that same animal had defecated $1.0 \mathrm{~g}$ during another interval, and the concentration during that interval was also $100 \mathrm{ng} / \mathrm{g}$, the total mass for that interval would be $100 \mathrm{ng}$ (see the Discussion for further details on methods of reporting steroid content).

\section{$R I A$}

A commercially available $\left[{ }^{125}\right.$ I]RIA (ICN Biomedical, Costa Mesa, CA, USA) for rat and mouse serum/plasma corticosterone was used to quantify fecal corticoids in both males and females. To ensure antibody binding along the linear portion of the standard curve ( 20 and $80 \%$ binding), fecal extracts were diluted 1:25 (or more) with diluent provided with the RIA kit. All samples were assayed in duplicate and re-analyzed if the coefficient of variation exceeded 10\%. Two control samples (each made by pooling fecal extracts from four or five animals) were analyzed in every assay (the 'low' pool with approximately $60 \%$ binding, and the 'high' pool with approximately $30 \%$ binding). Based on repeated analysis of these samples, intra- and inter-assay coefficients of variation for the assays of male samples were $5 \cdot 23 \%(n=5)$ and $9 \cdot 75 \%(n=6)$ for the low pool and $7 \cdot 22 \%(n=4)$ and $8 \cdot 35 \%(n=5)$ for the high pool. For the females, intra- and inter-assay coefficients of variation were $8 \cdot 83 \%(n=5)$ and $17 \cdot 81 \%$ $(n=5)$ for the low pool and $8 \cdot 00 \%(n=5)$ and $14 \cdot 22 \%$ $(n=8)$ for the high pool.

To determine the parallelism of diluted fecal extracts, a concentrated pooled extract made from nine individual fecal extracts was serially diluted (1:4 to $1: 64)$ and the slope of their antibody binding compared with that of the standards supplied with the RIA kit. To determine if the extract medium interfered with specific antibody binding, recoveries of known concentrations of corticosterone were calculated after assay in the presence of fecal extracts containing low concentrations of endogenous hormone.

Male fecal extracts were assayed within 1 month of extraction. Female extracts were analyzed approximately 12 months from initial extraction. To evaluate any possible decay in the samples during this storage period, we compared corticoid concentrations in 20 fecal extracts diluted for assay within a month of extraction and the same extracts stored for 12 months before dilution and assay. The correspondence between these repeated measures was excellent $\left(R^{2}=0.934\right)$, indicating a strong linear relationship. When we regressed results for the later analyzed samples on the immediately analyzed values, we found a slope of 1.045, indicating that high and low samples decayed at a similar rate over the delay period. Furthermore, an intercept of $60.10 \mathrm{ng} / \mathrm{g}$ indicated that the later-analyzed female samples, on average, were approximately $60 \mathrm{ng} / \mathrm{g}$ lower than if they had been analyzed immediately after extraction. This decay was significant $\left(t_{20}=4.68, P<0 \cdot 001\right)$ and was taken into account in the comparison of our male and female fecal corticoid values, which are presented as measured.

\section{Co-chromatography analysis of commercial antibody binding}

The number and relative proportions of immunoreactive fecal corticosteroid metabolites were determined by reverse-phase HPLC (Microsorb RP C-18; Rainen Instruments, Woburn, MA, USA) as described previously (Monfort et al. 1990). Before HPLC, fecal extracts were pre-processed using reverse-phase $\mathrm{C}_{18}$ cartridges (SpiceTM; Analtech, Newark, DE, USA; Heikkinen et al. 1981, Monfort et al. 1990). A $55 \mu$ portion of fecal extract, combined with ${ }^{3} \mathrm{H}$-cortisol (2500 d.p.m.) and ${ }^{3} \mathrm{H}$-corticosterone (2500 d.p.m.), was separated using a linear gradient of $20-100 \%$ methanol in water within $80 \mathrm{~min}(1 \mathrm{ml} / \mathrm{min}$ flow rate, $1.0 \mathrm{ml}$ fractions). Separate aliquots of each eluate were counted directly to determine recovery and/or assayed to identify immunoreactive corticosteroid metabolites.

\section{Identification of corticosterone metabolites}

To determine the corticosterone metabolites binding to the ICN antibody, pooled immunoreactive HPLC eluates were subject to LC-MS analysis. Six corticosterone metabolites were identified for detection based on established metabolic pathways in the rat: (1) 11-dehydro-

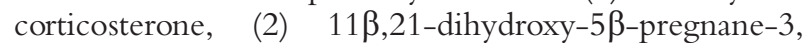
20-dione, (3) 21-hydroxy-5 $\beta$-pregnane-3,11,20-trione, 


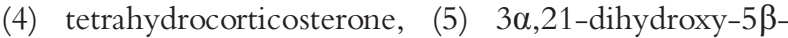

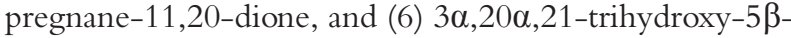
pregnane-11-one. Given that rats produce little or no cortisol (Bentley 1976), seven cortisol metabolites served as negative controls: (1) $11 \beta, 17 \alpha, 21$-trihydroxy-5 $\beta$ pregnane-3,20-dione, (2) urocortisol, (3) cortol, (4) cortisone, (5) 17 $\beta, 21$-dihyroxy-5 $\beta$-pregnane-3,11,20-trione, (6) urocortisone, and (7) cortolone.

For each sex, fractions 44-47 were analyzed because (a) these eluates represented the major immunoreactive peak, (b) they had similar polarity as immunoreactive peaks reported in other validation studies (Bamberg et al. 2001, Monfort et al. 1998), and (c) there was a clear sex difference in antibody binding to these eluates. Fractions 44-47 were combined and loaded on to a $\mathrm{C}_{18}$ cartridge (Waters Corporation, Milford, MA, USA). HPLC eluates that had been diluted with RIA diluent were dried down for transport, then reconstituted with methanol. To remove salt and impurities, samples were washed with loading buffer ( $5 \%$ methanol aqueous solution, containing $5 \mathrm{mM}$ sodium acetate and $0 \cdot 1 \%$ formic acid) at $2 \mu \mathrm{l} / \mathrm{min}$ flow rate, and then washed with elution buffer $(95 \%$ methanol aqueous solution, containing $5 \mathrm{mM}$ sodium acetate and $0 \cdot 1 \%$ formic acid) at $2 \mu \mathrm{l} / \mathrm{min}$ flow rate. This cleaning method was validated with standard samples containing $0 \cdot 5 \mathrm{pg}$ of eight standards: (1) equilin $(1,3,5[10]$, 7-estratetraen-3-ol-17-one), (2) 1,3,5[10]-estratrien-3ol-17-one, (3) 5 $\alpha$-androstan-17-one, (4) dehydroisoandrosterone (5-androsten-3 $\beta$-ol-17-one), (5) androsterone ( $5 \alpha$-androstan-3 $\alpha$-ol-17-one), (6) progesterone, (7) 11 dehydrocorticosterone (4-pregnen-21-ol-3,11,20-trione), and (8) corticosterone (4-pregnen-11ß,21-diol-3, 20dione).

\section{Statistical analyses}

To quantify the fecal corticoid circadian rhythm we calculated several characteristics of each animal's rhythm: peak value, trough value, acrophase, and nadir. In addition, to quantify the rate at which corticoid levels rise from trough to peak values and fall from peak to trough values we calculated the slopes of these functions (i.e. fecal corticoid value $=$ slope $\times$ time + intercept $)$. Slopes were calculated by regressing, for each animal, fecal corticoid values on time (in hours). Rising-slope calculations used all points from the trough to peak, falling-slope calculations included all points from the peak to the trough. To test the hypothesis that the rise in fecal corticoids occurs faster than the fall, we compared the absolute value of these two slopes within each individual with paired $t$-tests. Given the assymetrical (e.g. saw-toothed) circadian rhythm of circulating corticoids, the working hypothesis was that the slope of the rise would be steeper than the slope of the fall. Female fecal corticoid values (troughs, peaks, acrophase, nadir) were compared across days of the estrous cycle using repeated-measures ANOVAs. Male and female fecal corticoid values (troughs, peaks) were compared using $t$-tests.

\section{Results}

\section{Defecation profile of adult Sprague-Dawley rats}

The rats' defecation pattern permitted frequent daily sampling within individuals. Adult Sprague-Dawley rats defecated $46 \pm 2$ fecal pellets per day, with seven pellets (range, 0-16) defecated during each sampling period. The majority of pellets were defecated during the dark-phase sampling intervals $(10: 30,13: 30,16: 30$, and 19:30 h CDST), but a significant portion (35\%) was excreted during the light-phase sampling intervals (22:30, 3:00, and 7:30 h CDST). Across a complete day of fecal sampling (seven collection intervals), both sexes typically produced fecal pellets during six or seven intervals, and on average five or six samples were not contaminated with urine. The least-common intervals for defecation were at the beginning and end of the light phase (22:30 and 7:30 h).

Because water content can vary from animal to animal, fecal mass is expressed as dry weight. On average, male rats defecated $6 \cdot 88 \pm 0 \cdot 21 \mathrm{~g}$ per day and the females defecated $3 \cdot 96 \pm 0.18 \mathrm{~g}$ per day. Approximately $60 \%$ of the total daily fecal mass was excreted during the lights-off active dark phase $(10: 30,13: 30,16: 30,19: 30 \mathrm{~h}$ CDST). On average, the males defecated $1 \cdot 17 \pm 0.02 \mathrm{~g} / 3 \mathrm{~h}$ during the active phase and $0.72 \pm 0.18 \mathrm{~g} / 3 \mathrm{~h}$ during the lights-off inactive phase (22:30, 3:00, 7:30 CDST). Females showed a similar pattern, with an average of $0.65 \pm 0.03 \mathrm{~g} / 3 \mathrm{~h}$ during the active phase and $0.34 \pm 0.02 \mathrm{~g} / 3 \mathrm{~h}$ during the inactive phase. The sex difference in fecal weight was comparable to sex differences in body weight (females weigh approximately $60 \%$ that of males). Even during the less-productive inactive phase, fecal mass was adequate for quantifying fecal steroids for both male and female adult rats. To account for the quantitative difference in fecal mass across the day, we expressed the amount of fecal corticoid metabolites in feces as a total mass excreted per 3-h sampling period. Fecal mass and fecal corticoid concentration were not correlated either within or between individuals, supporting the working hypothesis that the amount of corticoid metabolites in bile is not associated with the production of fecal pellets.

\section{Biochemical validation of extraction and assay}

Recovery of radioactively labeled corticosterone during the extraction procedure was $90 \%(n=72)$. Serial dilutions of pooled rat fecal extracts for males and females yielded displacement curves parallel to standard corticosterone. Mean proportional recovery of added corticosterone (range, $0 \cdot 003-0 \cdot 125 \mathrm{ng}$ ) for the female pool was $99 \pm 6 \%$ $\left(y=0 \cdot 807 x+0 \cdot 667, R^{2}=0 \cdot 99\right)$, and for the male pool it was 


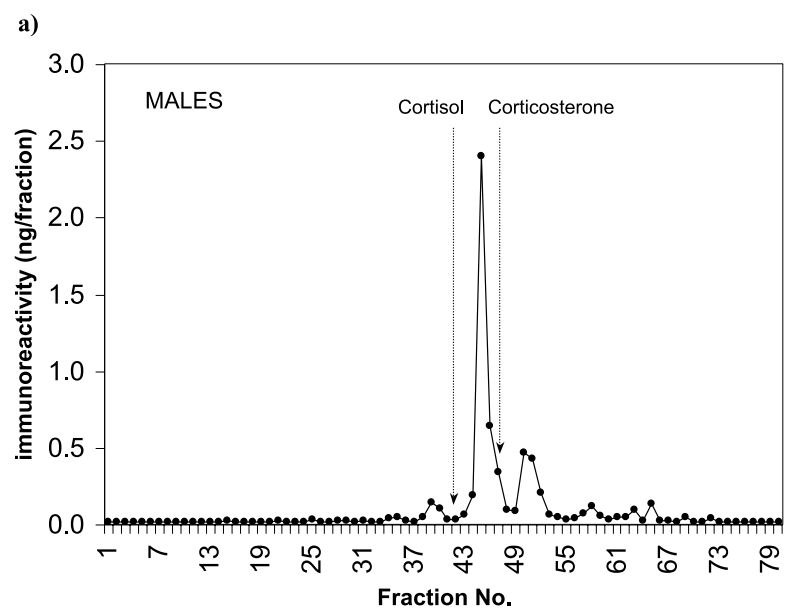

b)

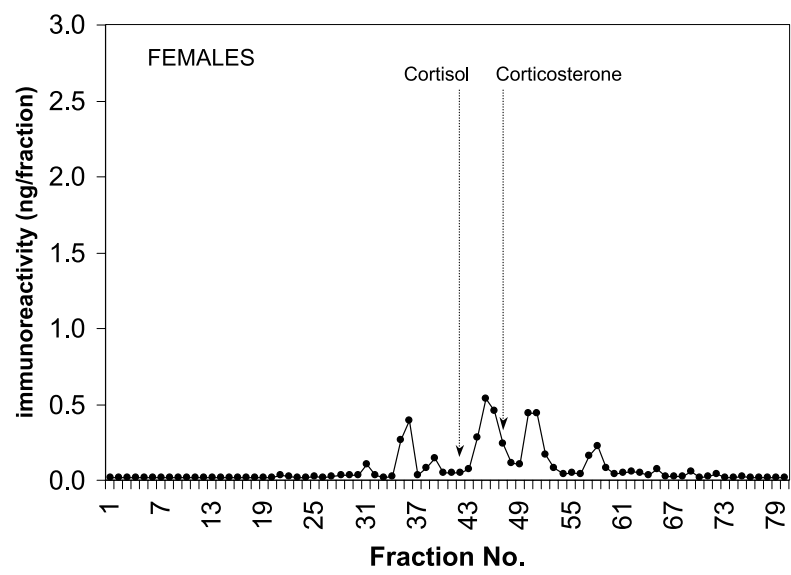

Figure 1 Immunoreactivity of (a) male and (b) female HPLC fractions to ICN corticosterone antibody.

$89 \pm 7 \%\left(y=0 \cdot 941 x+0 \cdot 199, R^{2}=0 \cdot 99\right)$. RIA of HPLCseparated fecal extracts from both males and females revealed a major immunoreactive peak that exhibited polarity intermediate between cortisol and corticosterone. Four additional minor immunoreactive peaks (two more polar than cortisol, and two less polar than corticosterone) were observed in both sexes (Fig. 1).

The averaged LC-MS spectra of male samples produced the following major ions: $331,341,351,362,373,385$, $333,335,337,359,310,367,369,315,397$, and 325 . Ions 373,367 , and 369 correspond to tetrahydrocorticosterone (or $3 \alpha, 20 \alpha, 21$-trihydroxy-5 $\beta$-pregnane-11-one), 11-dehydrocorticosterone, and 21-hydroxy-5 $\beta$-pregnane3,11,20-trione, respectively (Fig. 2). Fewer major ions were detected in the female sample: $362,333,373,310$, 335, 367, and 369. Again, ions 373, 367, and 369 correspond to tetrahydrocorticosterone (or $3 \alpha, 20 \alpha$, 21 -trihydroxy-5 $\beta$-pregnane-11-one), 11-dehydrocortico-

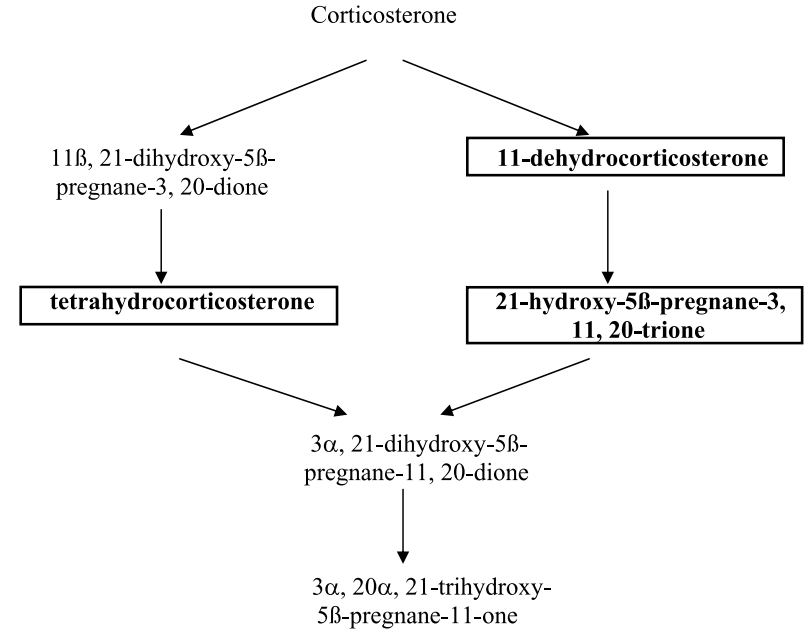

Figure 2 Metabolic pathways of corticosterone degradation in the rat. Boxes indicate metabolites (identified by LC-MS) in the most immunoreactive HPLC fractions (Fig. 1, fractions 44-47).

sterone, and 21-hydroxy-5 $\beta$-pregnane-3,11,20-trione, respectively. Cortisol metabolites were not present in the analyzed eluates, nor were the remaining three corticosterone metabolites: 11 $\beta, 21$-dihydroxy- $5 \beta$-pregnane3,20-dione, $3 \alpha, 21$-dihydroxy- $5 \beta$-pregnane-11,20-dione, and $3 \alpha, 20 \alpha, 21$-trihydroxy- $5 \beta$-pregnane-11-one.

\section{Adjusting for urine contamination}

Because rats excrete only $20 \%$ of corticoids in urine (Bamberg et al. 2001), it is possible that fecal samples soaked by urine may have their corticoids leached or diluted, leading to lowered fecal corticoid levels. Indeed, urine contamination did decrease fecal corticoid levels. When comparing samples contaminated and uncontaminated by urine, collected from the same individuals at the same time of day, contaminated samples had lower fecal corticoid values than uncontaminated samples (e.g. from 10:30 h collection: $110 \cdot 4$ versus $135.5 \mathrm{ng} / 3 \mathrm{~h} ; t_{9}=2 \cdot 60$, $P<0 \cdot 05)$.

Approximately one-quarter of the male samples and one-tenth of the female samples were found on a patch of urine-soaked bedding (identified as urine-contaminated or wet). We determined how much metabolites were leached by urine to adjust the corticoid values obtained from these wet samples. In 35 cases among the males, separately stored wet and dry (i.e. not urine-contaminated) samples were available for the same animal from the same collection period. To determine the relationship between wet and dry samples, we regressed the natural logarithm of the corticoid concentration for the dry samples on the logarithm of the concentration for the corresponding wet samples. The estimated intercept was 1.43 (S.E.M. $=0 \cdot 38$ ) and the estimated slope was $0 \cdot 76$ (S.E.M. $=0 \cdot 07$ ). A test of 


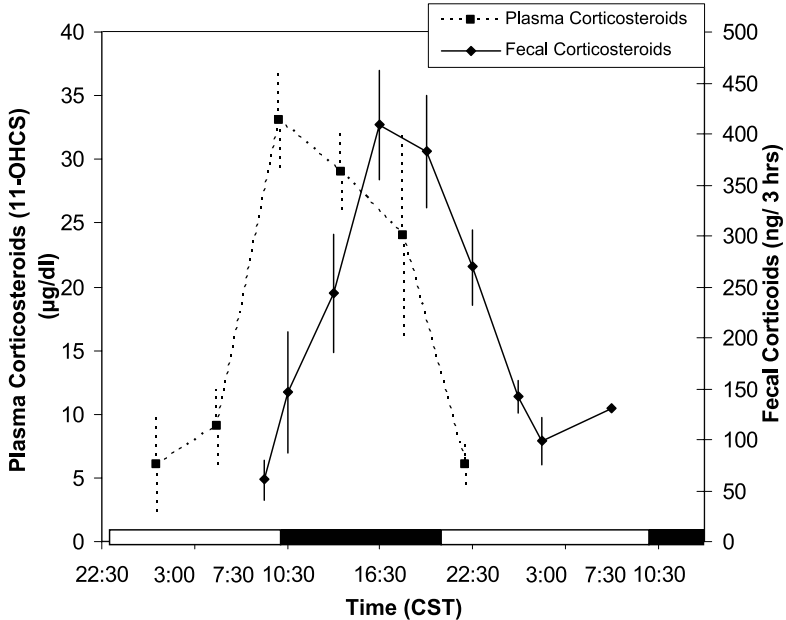

Figure 3 Mean ( \pm S.E.M.) fecal corticoid values (solid line) at 3-h intervals for nine adult male Sprague-Dawley rats during a 24-h period, compared with previously published values of male and female circulating corticosteroid rhythm (dashed line) from Krieger (1973). Bars indicate S.E.M. The black areas on the abscissa indicate dark phases of the lighting cycle (the behavioral day in this nocturnal species).

the hypothesis that the true slope is equal to 1 (corresponding to a proportional relationship between the corticoid values for wet and dry samples) yielded a $P$ value of 0.002 . The $R^{2}$ value was $0 \cdot 78$, and a plot of the residuals indicated that the linear model fitted the data well. This estimated model was used to adjust corticoid concentrations in wet samples for both the males and females.

\section{Fecal corticoid circadian rhythm in males}

Males exhibited a distinct circadian rhythm in fecal corticoid excretion (Fig. 3). Peak corticoid levels occurred at the end of the active period and were approximately seven times greater than trough levels $(512.6 \pm 31 \cdot 4$ versus $74 \cdot 5 \pm 9 \cdot 0 \mathrm{ng} / 3 \mathrm{~h}$ ). The acrophase typically occurred during the $16: 30$ or 19:30 h intervals, corresponding to the end of the dark phase, approximately 6-9 h from the normal peak in circulating corticoids (Krieger 1973). Nine of the 10 males had their fecal corticoid acrophase during these two collection intervals. The nadir in fecal corticoid excretion generally occurred during the $3: 00$ or $7: 30 \mathrm{~h}$ interval, corresponding to the middle and end of the light phase. Six of the 10 males excreted their lowest corticoid levels during these two intervals.

Males excreted corticoids in an asymmetrical (sawtoothed) circadian rhythm (Figs 3 and 4). Fecal corticoid concentrations in males rose from lowest to highest values within $9 \mathrm{~h}$, with seven out of 10 males following this pattern. In contrast, it took approximately $15 \mathrm{~h}$ (or five collection intervals) for fecal corticoids to fall to nadir (a)

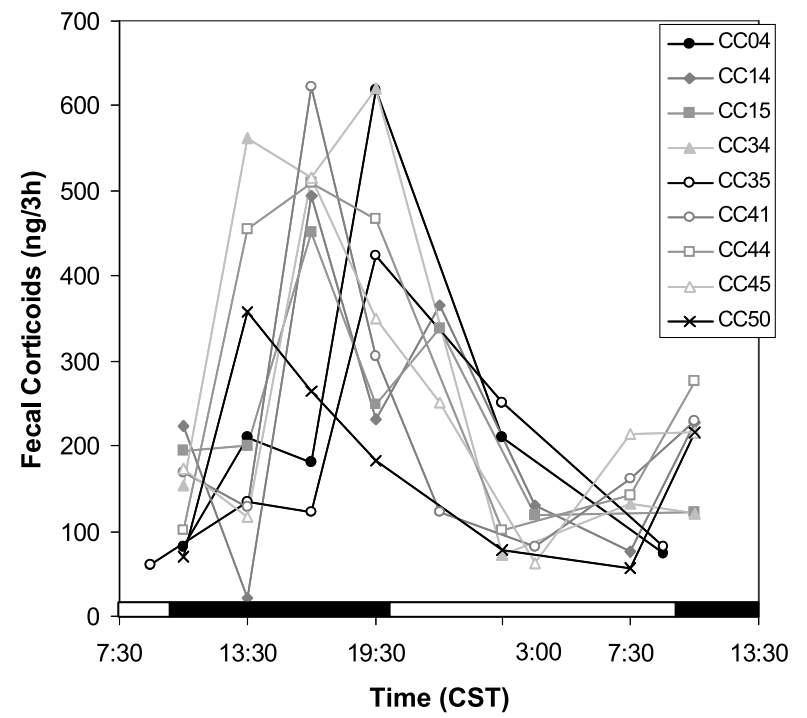

(b)

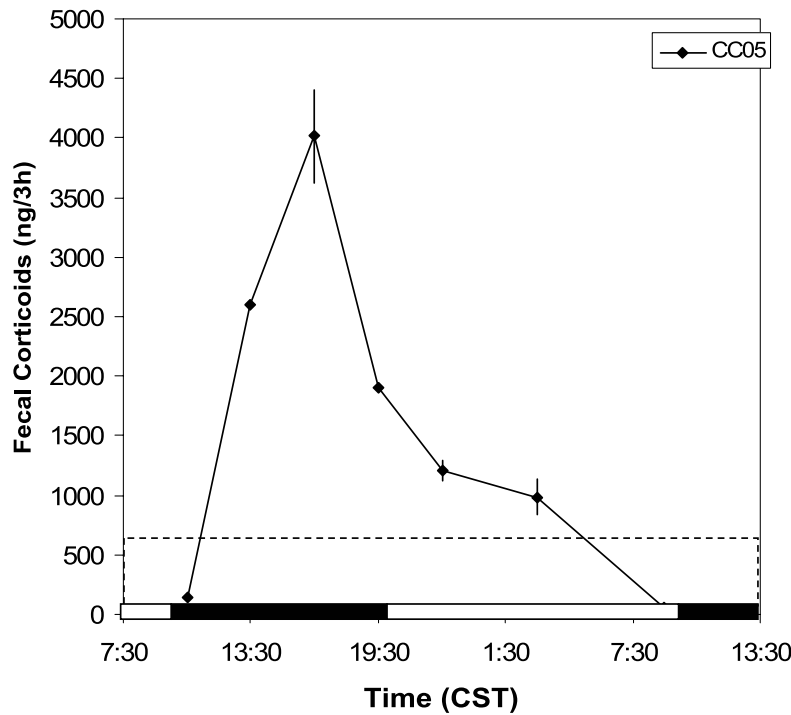

Figure 4 (a) Individual adult male fecal corticoid values every 3-6 h over a 24-h period. Black bars on the abscissa indicate dark phases of the lighting cycle (rats' active period). (b) Male CC05 fecal corticoid values over $24-\mathrm{h}$ period. The horizontal dashed line indicates the upper limit of values for the nine other males plotted in (a). Error bars indicate S.E.M. from repeated analyses of the same sample collected from this male.

concentrations. Thus, fecal corticoid levels rose from nadir to the peak in $6 \mathrm{~h}$, less time than it took to decline from the peak to nadir levels (i.e. fecal corticoid values increased at a rate of approximately $12 \mathrm{ng} / \mathrm{h}$ and declined at a rate of approximately $8 \mathrm{ng} / \mathrm{h}$ ). The absolute value of the 
ascending slope was significantly greater than the absolute value of the descending slope $\left(t_{8}=2 \cdot 25, P<0 \cdot 05\right)$.

Differences in fecal corticoid excretion were quite marked among different individual males. The 24-h mean fecal corticoid value for one male was $>30$ standard deviations higher than for any other male (Fig. 4b). This male was excluded from the analysis of peaks, troughs, and slopes presented above because its corticoid concentrations were so dramatically atypical. Interestingly, this male was also behaviorally distinct from the other males: it was consistently slower to emerge from a familiar setting and slower to explore a complicated novel environment (S.A. Cavigelli \& M.K. McClintock, unpublished observations). The average peak corticoid value for the nine males was $512.6 \mathrm{ng} / 3 \mathrm{~h}$, whereas the peak corticoid value for the excluded male (Fig. 4b) was 6-fold higher than the next highest male (peak value, $4014 \cdot 3 \mathrm{ng} / 3 \mathrm{~h}$ ).

\section{Fecal corticoid circadian rhythm in females}

Fecal corticoids in females also were excreted in a circadian pattern, with peak values 6-18 times higher than trough values. However, key elements of this rhythm (e.g. peak and trough values) varied with the day of the estrous cycle (Fig. 5, upper panel). Daily corticoid means were lowest on the day of estrus and rose progressively during metestrus, diestrus, and proestrus $\left(\mathrm{F}_{3,8}=10 \cdot 90, P<0 \cdot 0001\right)$. Peak fecal corticoid levels on proestrus were two or three times higher than peaks during estrus or metestrus. Peak corticoid concentrations were lowest during estrus and sequentially higher during metestrus, diestrus, and proestrus $\left(\mathrm{F}_{3,8}=6 \cdot 44, P<0 \cdot 01\right)$. Average trough values were not significantly different across the days of the estrous cycle $\left(\mathrm{F}_{3,8}=0 \cdot 53, P=0 \cdot 66\right)$.

Throughout the estrous cycle, the acrophase and nadir of fecal corticoids for females were approximately 16:30 h and 7:30 h, respectively. As with the males, the majority of female acrophases $(30 / 36)$ were in the $16: 30$ or $19: 30 \mathrm{~h}$ intervals, corresponding to the end of the dark phase. The majority of nadirs $(28 / 36)$ were during the same intervals as the males: the 3:00 and 7:30 h intervals, i.e. middle to end of the lights-on period.

\section{Sex differences}

On average, males excreted more immunoreactive fecal corticoids than did females. The daily mean fecal corticoid produced in males was greater than the female mean on all days of the estrous cycle $\left(t_{43}=14 \cdot 23, P<0 \cdot 0001\right)$. Male trough and peak levels were repeatedly greater than female trough and peak levels (trough, $t_{43}=12.92, P<0 \cdot 0001$; peak, $\left.t_{43}=9 \cdot 19, P<0 \cdot 0001\right)$. These differences did not disappear when females samples were adjusted to account for the storage decay (daily mean, $t_{43}=13 \cdot 39, P<0 \cdot 0001$; trough, $\quad t_{43}=11 \cdot 25, \quad P<0 \cdot 0001 ; \quad$ peak, $t_{43}=8 \cdot 04$,
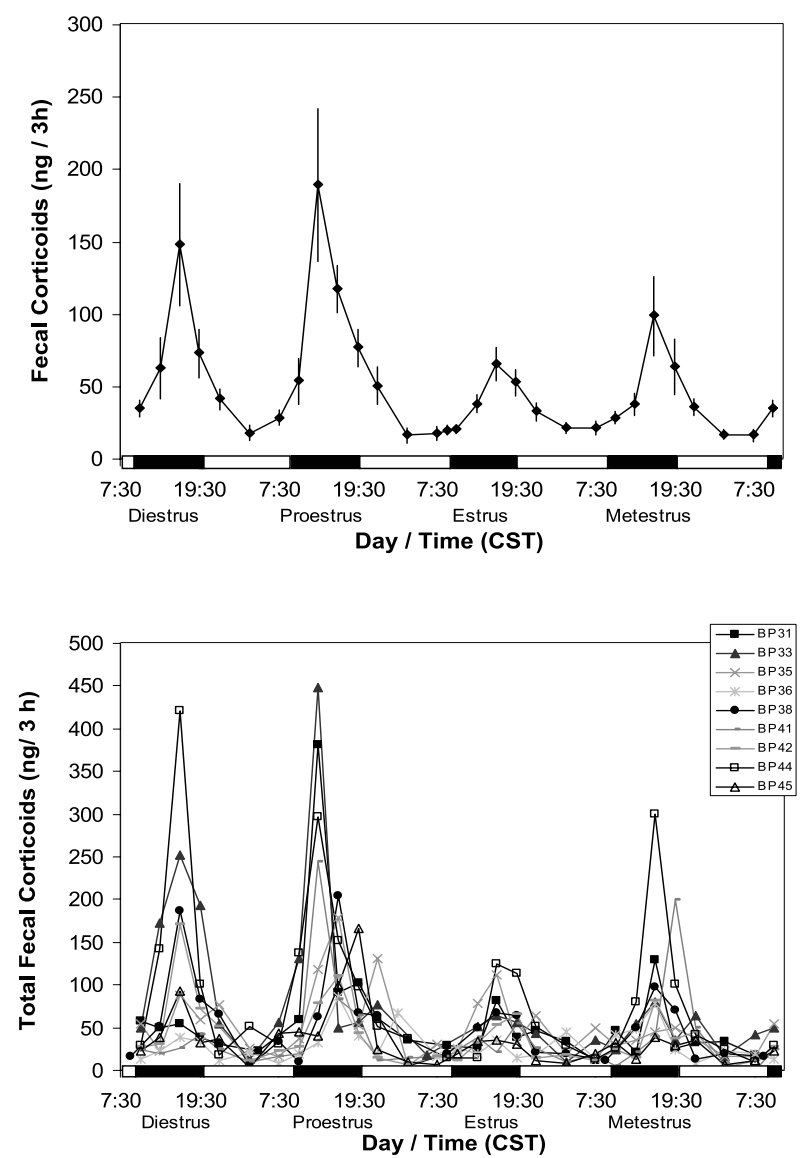

Figure 5 Upper panel: mean ( \pm S.E.M.) fecal corticoid values over the 4-day estrous cycle for adult female rats. Black bars on the abscissa indicate dark phases of the lighting schedule (rats' active period). Lower panel: individual fecal corticoid values over a 4-day period for nine adult female Sprague-Dawley rats.

$P<0 \cdot 0001)$, even when analyses were limited to proestrus, the day when females' corticoid values were highest (daily mean, $t_{16}=7 \cdot 58, P<0 \cdot 0001$; trough, $t_{16}=6 \cdot 22, P<0 \cdot 0001$; peak, $\left.t_{16}=4 \cdot 83, P<0 \cdot 001\right)$. There were no differences between male and female acrophases or nadirs (acrophase, $t_{43}=0.91, P=0.37$; nadir, $t_{43}=0.73, P=0 \cdot 47$ ). For both the males and females, the shape of the circadian rhythm was similar, with a rapid rise in levels in $9 \mathrm{~h}$ and a slower return to trough levels in approximately $15 \mathrm{~h}$ (Figs 4 and 5). Finally, females produced fewer immunoreactive corticoids in the major HPLC-immunoreactive fractions (44-47; Fig. 1), and they had fewer major ions in these fractions compared with males.

\section{Discussion}

Fecal corticoids were excreted in a clear circadian rhythm with well-defined acrophases and nadirs. Sampling at 
3-h intervals provided a high degree of temporal acuity for characterizing the circadian corticoid rhythm. Undisturbed male Sprague-Dawley rats excreted fecal corticoids in an asymmetrical pattern consisting of a rapid rise in corticoid excretion followed by a more gradual return to trough levels over the day. This function was not a by-product of averaging among many individuals, but was present within each individual. This asymmetrical rhythm was similar to that identified for circulating corticosteroids in rats (Krieger 1973) and humans (Dahl et al. 1991, Frank et al. 1995, Rohatagi et al. 1996).

Technical limitations associated with repeated blood sampling from small rodents over time likely hinder studies designed to evaluate the function and mechanisms of the circadian corticoid rhythm. The fecal corticoid measures provide an interesting complement to blood measures because feces can be collected with minimal animal disturbance over extended intervals without altering circadian rhythms - a concern that must be addressed when employing a chronic in-dwelling catheter. The asymmetrical excretory rhythms were consistent among animals, suggesting this method can be used for tracking circadian-rhythm alterations in individual animals. Finally, these results call into question the use of the cosinor analyses for estimating the corticoid circadian rhythms in rats (e.g. Atkinson \& Waddell 1997). Our data suggest that more accurate methods for modeling this circadian rhythm need to be developed (e.g. Rohatagi et al. 1996, Wang \& Brown 1996, Brown et al. 2001).

The amplitude of the fecal circadian corticoid rhythm is very similar to that for circulating corticosterone in Sprague-Dawley rats (Krieger 1973). In males, the peakto-trough ratio of fecal corticoids is approximately 7:1 and the ratio for circulating corticosterone is approximately 10:1 (Krieger 1973). Given that fecal corticoid levels represent an integration of circulating corticoid levels, it might be surprising that the fecal corticoid amplitude does not show greater dampening relative to the circulating corticosterone amplitude. This lack of dampening, however, is likely due to the rats' high defecation rate within a day. The sampling density enables high temporal resolution with six or seven samples per day per animal. The integration of steroids in feces occurs only over relatively short intervals ranging from 3 to $4 \mathrm{~h}$. This frequent, short-term temporal integration may be a distinct advantage for those interested in using a non-invasive method to document corticoid circadian rhythms or corticoid levels over extended periods.

The daily acrophase and nadir for fecal corticoid excretion occurred approximately 6-9 h later than the known acrophase and nadir for circulating corticosterone levels (Krieger 1973, Atkinson \& Waddell 1997). This same lag time was true for both males and females and is similar to other time lags determined from studies that exogenously stimulated an elevation in circulating gluco- corticoids (Bamberg et al. 2001, 12-18 h lag time; Touma et al. 2003, 4-8 h lag time; Royo et al. 2004, $12 \mathrm{~h} \mathrm{lag}$ time). It should be noted that previously identified time lags greater than $9 \mathrm{~h}$ were found in studies that used infrequent sampling intervals (e.g. every $12 \mathrm{~h}$ ), which gives greater credence to the $6-9 \mathrm{~h}$ lag period. By using the naturally occurring circadian rhythm to estimate the time lag between circulating and excreted corticoids, we avoided animal handling (i.e. required for exogenous corticotropin or corticosteroid administration), which has the potential to alter excretory lag time via sympathetic nervous system effects on gastrointestinal transit time. A well-defined time lag (6 to $9 \mathrm{~h}$ ) provides another unique advantage to using fecal corticoid measures. This time lag permits researchers to assess adrenal responses to experimental manipulations after the manipulation has occurred, rather than disrupting the procedure to collect contemporaneous blood samples. Physiological responses to environmental stimuli or stressors can be evaluated well after the interaction of interest with a precise and integrated measure of adrenal activity.

Corticoid metabolites in male and female SpragueDawley rat fecal extracts were both more and less polar than corticosterone. The HPLC co-chromatography profile for the rat fecal extracts was comparable to other reports in rodents and other species (Bamberg et al. 2001, Monfort et al. 1998) in which the same ICN corticosterone antibody was used. This commercial antibody used in the present study is known to cross-react with a variety of corticosteroid metabolites, and is therefore referred to as a group-specific antibody that appears to be particularly sensitive to changes at sites C-11 and C-21 (Wasser et al. 2000). LC-MS analyses of the major immunoreactive HPLC eluates verified that the ICN antibody detects at least three of the six major corticosterone metabolites in rat feces (tetrahydrocorticosterone, 11-dehydrocorticosterone, and 21-hydroxy-5 $\beta$-pregnane-3,11,20-trione). The remaining three metabolites may have been (1) present in untested eluates, (2) present in mass quantities that were below detection by either immunoassay of HPLC co-chromatography or LC-MS in tested eluates, or (3) metabolized to non-immunoreactive forms. Further studies are planned to address these questions. The laboratory validation clearly reveals that we can detect a group of fecal corticosterone metabolites, some of which are major metabolites of corticosterone. While unmetabolized corticosterone is not present in rat excreta, we have demonstrated the physiological validity of measuring a group of fecal corticoid metabolites to track adrenal rhythms in both sexes of the rat.

Co-chromatographic analysis revealed that the major immunoreactive metabolites detected by the ICN antibody had similar retention times for both sexes, although the degree of immunoreactivity for each fraction differed between the sexes. For example, the fraction of intermediate polarity between cortisol and corticosterone, 
containing the three identified metabolites, represented the majority of binding for males but not for females. In addition, males excreted greater concentrations of immunoreactive corticoids than females, which is interesting because females are known to secrete more corticosterone than males (e.g. Atkinson \& Waddell 1997). Sex-related differences in fecal corticoid concentrations may be partially explained by differences in steroid biosynthesis or catabolic pathways (Eriksson \& Gustafsson 1970). Females may excrete less overall mass of corticoid metabolites from blood into feces than males because their plasma corticosterone-binding capacity exceeds that of males, and they have a slower fractional clearance rate than males (Ottenweller et al. 1979, Woodward et al. 1991). Thus, females may catabolize less corticosterone through the liver and/or excrete less mass of corticosterone metabolites in feces, and more in urine, than males. Further studies on steroid metabolism and/or with alternative antibodies may help elucidate these sex differences. Nonetheless, between-sex similarities in the rhythms of fecal corticoid excretion (i.e. acrophase and nadir) were clear, which confirms the usefulness of fecal measures evaluating adrenal status in both sexes, and the multiple immunoreactive peaks suggest that the commercial antibody has the potential to respond to many corticosterone metabolites.

Fecal steroid measures are most frequently presented as concentrations (e.g. ng/g of feces). However, total mass of corticoids in the feces is a more accurate measure of production than relative concentration. Concentration of corticosterone in blood correlates highly with corticosterone production from the adrenal because blood volume is relatively constant. In feces, corticosterone from the blood is metabolized by the liver and enters the small intestines through the bile duct, independently of fecal mass, which is regulated throughout the small and large intestine (Schwarzenberger et al. 1996). Unlike blood, however, the volume (mass) of fecal matter defecated can change significantly both within and between days. For example, during the inactive portion of the day, rats produce less fecal mass than during active periods. This reduced fecal mass during inactivity provides less 'diluent' or 'medium' for the metabolized corticosterone passing through the bile duct, potentially resulting in 'falsely" elevated concentration of corticoids in feces during inactive periods.

When corticoid values are presented as concentrations per gram of fecal matter, the same amount of corticoids metabolized and secreted in the bile during an inactive and active period leads to very different reported concentrations: higher concentrations are reported during the inactive period when fecal volume is reduced, due to the decreased volume of fecal 'diluent'. (When we analyzed the data as corticoid concentration per gram of fecal matter, corticoid values were greater than expected during the inactive low-fecal-defecation periods (data not presented).)
Thus, reporting total fecal corticoids (as opposed to concentration) excreted over a given time period provides a more accurate index of corticoid production and blood concentration than does a fecal concentration value which assumes constant fecal ('diluent') volume; this is particularly true when frequency of defecation and total mass of feces are known for the study animals. We recommend this format for reporting fecal steroid results in future studies.

Non-invasive fecal measures have generally been used to assess steroid excretion in wild animal populations (e.g. Creel et al. 1997, Cavigelli 1999, Wasser et al. 2000, Goymann et al. 2001). However, it is clear that these same methods have tremendous potential, and a number of important advantages for studying laboratory animals, including that (1) behavior is not disturbed by sampling, (2) the circadian glucocorticoid rhythm is not affected by the possible stress associated with handling, restraint, and blood sampling, (3) sampling frequency is limited only by defecation rates, (4) individual animals can be monitored for days, months, and throughout life, which permits manipulation and documentation of long-term endocrine rhythms, (5) fecal measures represent a complete and integrated measure of hormone production because hormones are 'pooled' in the gut before excretion, and (6) fecal steroids may reflect unbound (i.e. the biologically active portion) circulating glucocorticoid titers, since bound steroids are less readily metabolized by the liver (Westphal 1971, 1983, Leeper et al. 1988).

We demonstrated the utility of fecal corticoid measures for assessing the circadian corticosteroid rhythm and for assessing long-term changes in corticoid production in individual laboratory rats. This method may also have applications in other small laboratory animals that possess high metabolic and frequent defecation rates, characteristics that facilitate frequent, repeated non-invasive fecal sample collection. Among the most compelling uses of this method will be for conducting long-term studies to understand how external factors modulate circadian rhythmicity and/or produce chronic changes in corticoid production in laboratory rats.

\section{Acknowledgments}

We thank Phil Schumm for his assistance in regression analyses of urine contamination. The research was supported by NICHD F32 HD08693 (to S A C), NIMH R37 MH41788 (to M K M), and NIA PO1 AG018911 (to $\mathrm{M} \mathrm{K} \mathrm{M}$ ). The MS analysis was supported by the Indiana Genomics Initiative (INGEN), which is funded in part by the Lilly Endowment. The authors declare that there is no conflict of interest that would prejudice the impartiality of this scientific work. 


\section{References}

Atkinson HC \& Waddell BJ 1997 Circadian variation in basal plasma corticosterone and adrenocorticotropin in the rat: sexual dimorphism and changes across the estrous cycle. Endocrinology 138 3842-3848.

Bamberg E, Palme R \& Meingassner JG 2001 Excretion of corticosteroid metabolites in urine and faeces of rats. Laboratory Animals 35 307-314.

Bentley PJ 1976 Comparative Vertebrate Endocrinology, p 67. New York: Cambridge University Press.

Brown EN, Meehan PM \& Dempster AP 2001 A stochastic differential equation model of diurnal cortisol patterns. American Journal of Physiology Endocrinology and Metabolism 280 E450-E461.

van Cauter E, Leproult R \& Kupfer DJ 1996 Effects of gender and age on the levels and circadian rhythmicity of plasma cortisol. Journal of Clinical Endocrinology and Metabolism 81 2468-2473.

Cavigelli SA 1999 Behavioural patterns associated with faecal cortisol levels in free-ranging female ring-tailed lemurs, Lemur catta. Animal Behaviour 57 935-944.

Creel S, Creel NM, Mills MGL \& Monfort SL 1997 Rank and reproduction in cooperatively breeding African wild dogs: behavioral and endocrine correlates. Behavioral Ecology 8 298-306.

Dahl RE, Ryan ND, Puig-Antich J, Nguyen NA, Al-Shabbout M, Meyer VA \& Perel J 1991 24-hour cortisol measures in adolescents with major depression: a controlled study. Biological Psychiatry 30 25-36.

Eriksson H \& Gustafsson J 1970 Steroids in germfree and conventional rats: distribution and excretion of labeled pregnenolone and corticosterone in male and female rats. European Journal of Biochemistry 15 132-139.

Frank S, Roland DC, Sturis J, Byrne MM, Spire JP, Refetoff S, Polonsky KS \& van Cauter E 1995 Effects of aging on glucose regulation during wakefulness and sleep. American Journal of Physiology Endocrinology and Metabolism 269 E1006-E1016.

Gala RR \& Westphal U 1965 Corticosteroid-binding globulin in rat studies on sex difference. Endocrinology 77 841-851.

Gans SE \& McClintock MK 1993 Individual differences among female rats in the timing of the preovulatory LH surge are predicted by lordosis reflex intensity. Hormones and Behavior 27 403-417.

Goymann W, Möstl E, Van't Hof T, East ML \& Hofer H 1999 Noninvasive fecal monitoring of glucocorticoids in spotted hyenas, Crocuta crocuta. General and Comparative Endocrinology 114 340-348.

Goymann W, East ML, Wachter B, Höner OP, Möstl E, Van't Hof J \& Hofer H 2001 Social, state-dependent and environmental modulation of faecal corticosteroid levels in free-ranging female spotted hyenas. Proceedings of the Royal Society of London, Series B $2682453-2459$.

Guilleman R, Dean E \& Liebelt RA 1959 Nychthemeral variations in plasma free corticosteroid level of the rat. Proceedings of the Society for Experimental Biology and Medicine 101 394-395.

Haemisch A, Guerra G \& Furkert J 1999 Adaptation of corticosterone - but not beta-endorphin - secretion to repeated blood sampling in rats. Laboratory Animals 33 185-191.

Harper JM \& Austad SN 2000 Fecal glucocorticoids: a noninvasive method of measuring adrenal activity in wild and captive rodents. Physiological and Biochemical Zoology 73 12-22.

Heikkinen R, Fotsis T \& Adlercreutz H 1981 Reversed-phase C18 cartridge for extraction of estrogens from urine and plasma. Clinical Chemistry 27 1186-1189.

Kern W, Dodt C, Born J \& Fehm HL 1996 Changes in cortisol and growth hormone secretion during nocturnal sleep in the course of aging. Journal of Gerontology 51A, M3-M9.

Kishibayashi N, Yokoyama T \& Karasawa A 1995 Enhancement of defecation and distal colonic motor activity by KW-5092, a novel gastroprokinetic agent, in rats. Archives Internationales de Pharmacodynamie et de Therapie 329 295-306.
Krieger DT 1973 Effect of ocular enucleation and altered lighting regimens at various ages on the circadian periodicity of plasma corticosteroid levels in the rat. Endocrinology 93 1077-1091.

Leeper LL, Schroeder R \& Henning SJ 1988 Kinetics of circulating corticosterone in infant rats. Pediatrics Research 24 595-599.

LeFevre J \& McClintock MK 1988 Reproductive senescence in female rats: a longitudinal study of individual differences in estrous cycles and behavior. Biology of Reproduction 38 780-789.

Linkowski P, Mendlewicz J, Leclerc R, Brasseur M, Hubain P, Golstein J, Copinschi G \& van Cauter E 1985 The 24-hour profile of adrenocorticotropin and cortisol in major depressive illness. Journal of Clinical Endocrinology and Metabolism 61 429-438.

Monfort SL, Arthur NP \& Wildt DE 1990 Monitoring ovarian function and pregnancy by evaluating excretion of urinary oestrogen conjugates in semi-free-ranging Przewalski's horses (Equus przewalskii). Journal of Reproduction and Fertility 91 155-164.

Monfort SL, Mashburn KL, Brewer BA \& Creel SR 1998 Evaluating adrenal activity in African wild dogs (Lycaon pictus) by fecal corticosteroid analysis. Journal of Zoo and Wildlife Medicine 29 129-133.

Ottenweller JE, Meier AH, Russo AC \& Frenke ME 1979 Circadian rhythms of plasma corticosterone binding activity in the rat and the mouse. Acta Endocrinologica 91 150-157.

Pfohl B, Sherman B, Schlechte J \& Stone R 1985 Pituitary-adrenal axis rhythm disturbances in psychiatric depression. Archives of General Psychiatry 42 897-903.

Ponzio MF, Monfort SL, Busso JM, Dabbene VG, Ruiz RD \& De Cuneo MF 2004 A non-invasive method for assessing adrenal activity in the chinchilla (Chinchilla lanigera). Journal of Experimental Zoology 301A, 218-227.

Retiene K, Zimmerman E, Schindler WJ, Neuenschwander J \& Lipscomb HS 1968 A correlative study of endocrine rhythms in rats. Acta Endocrinologica 57 615-622.

Rohatagi S, Bye A, Mackie AE \& Derendorf H 1996 Mathematical modeling of cortisol circadian rhythm and cortisol suppression. European Journal of Pharmaceutical Sciences 4 341-350.

Royo F, Bjork N, Carlsson HE, Mayo S \& Hau J 2004 Impact of chronic catheterization and automated blood sample (Accusampler) on serum corticosterone and fecal immunoreactive corticosterone metabolites and immunoglobulin A in male rats. Journal of Endocrinology 180 145-153.

Sachar EJ, Hellman L, Roffwarg HP, Halpern FS, Fukushim DK \& Gallaghe TF 1973 Disrupted 24-hour patterns of cortisol secretion in psychotic depression. Archives of General Psychiatry 28 19-24.

Schwarzenberger F, Möstl E, Palme R \& Bamberg E 1996 Faecal steroid analysis for non-invasive monitoring of reproductive status in farm, wild and zoo animals. Animal Reproduction Science 42 515-526.

Smith SW \& Gala RR 1977 Influence of restraint on plasma prolactin and corticosterone in female rats. Journal of Endocrinology $\mathbf{7 4}$ 303-314.

Spiegel K, Leproult R \& van Cauter E 1999 Impact of sleep debt on metabolic and endocrine function. Lancet 354 1435-1439.

Touma C, Sascher N, Möstl E \& Palme R 2003 Effects of sex and time of day on metabolism and excretion of corticosterone in urine and feces of mice. General and Comparative Endocrinology 130 267-278.

Tuli JS, Smith JA \& Morton DB 1995 Corticosterone, adrenal and spleen weight in mice after tail bleeding, and its effect on nearby animals. Laboratory Animals 29 90-95.

Wang Y \& Brown MB 1996 A flexible model for human circadian rhythms. Biometrics 52 588-596.

Wasser SK, Monfort SL, Southers J \& Wildt DE 1994 Excretion rates and metabolites of oestradiol and progesterone in baboon (Papio cynocephalus cynocephalus) faeces. Journal of Reproduction and Fertility 101 213-220.

Wasser SK, Hunt KE, Brown JL, Cooper K, Crockett CM, Bechert U, Millspaugh JJ, Larson S \& Monfort SL 2000 A generalized fecal 
glucocorticoid assay for use in a diverse array of nondomestic mammalian and avian species. General and Comparative Endocrinology $120260-275$

Westphal U 1971 Steroid-Protein Interactions, p 435. New York: Springer-Verlag.

Westphal U 1983 Steroid-protein interaction: from past to present.

Journal of Steroid Biochemistry 19 1-15.
Woodward CJH, Hervey GR, Oakey RE \& Whitaker EM 1991 The effects of fasting on plasma corticosterone kinetics in rats. Journal of Nutrition 66 117-127.

Received 26 July 2004

Accepted 15 October 2004 